\title{
Toward clinical equipoise: the current case for carotid angioplasty and stent placement
}

\author{
Elad I. LeVy, M.D., Robert D. Ecker, M.D., JAMEs J. Thompson, D.O., \\ Peter A. Rosella, M.D., Ricardo A. Hanel, M.D., Lee R. Guterman, Ph.D., M.D., \\ AND L. NELSON HopKINS, M.D.
}

Department of Neurosurgery and Toshiba Stroke Research Center, and Department of Radiology, School of Medicine and Biomedical Sciences, University at Buffalo, State University of New York, Buffalo, New York

\begin{abstract}
Recent advances in carotid artery (CA) stent placement procedures have propelled this technology into the forefront of treatment options for both symptomatic and asymptomatic patients with CA stenosis. Until recently, endarterectomy was the only surgical option for patients with CA occlusive disease. For high-risk surgical candidates, periprocedural stroke rates remained unacceptable and were significantly higher than those associated with the natural history of the disease. Advances in stent technology and improvements in antiplatelet and antithrombotic regimens, in conjunction with distal protection devices, have significantly lowered the risk of periprocedural complications for highrisk surgical candidates requiring CA revascularization. In this paper the authors review data gleaned from the important recent CA stent trials and address questions concerning the safety, efficacy, and durability of stent-assisted angioplasty for extracranial CA occlusive disease. Additionally, they review the role of noninvasive imaging modalities for the diagnosis and surveillance of CA disease in these high-risk patients.
\end{abstract}

\section{KEY WORDS - carotid artery stenosis - carotid artery stent • distal embolic protection}

\section{HISTORICAL BACKGROUND}

Carotid artery angioplasty with stent implantation dates back approximately 20 years. Catheter dilation of proximal CA stenosis during CEA was first reported by Kerber, et al. ${ }^{16}$ in 1980. Shortly thereafter, Bockenheimer and Mathias ${ }^{3}$ reported a series of three patients treated with percutaneous angioplasty. More than a decade would pass before reports of CA stent series emerged. In one such report, Bergeron, et al., ${ }^{2}$ described a $33 \%$ neurological complication rate with one death caused by hyperperfusion syndrome. The first randomized prospective clinical trial in which stent placement and surgery were compared was halted because of the significantly higher rate of complications in patients treated with stents. ${ }^{21}$ Five of seven patients in whom stents were placed experienced neuro-

Abbreviations used in this paper: BEACH = Boston Scientific EPI. A Carotid Stenting Trial for High-Risk Surgical Patients; $\mathrm{CA}=$ carotid artery CABERNET $=$ Carotid Artery Revascularization Using the Boston Scientific FilterWire EX/EZ and the EndoTex NexStent Trial; $\mathrm{CCA}=$ common CA; CEA = carotid endarterectomy; CREST = Carotid Revascularization Endarterectomy versus Stent Trial; CT = computerized tomography; DS = digital subtraction; $\mathrm{ICA}=$ internal $\mathrm{CA} ; \mathrm{MI}=$ myocardial infarction; $\mathrm{MR}=$ magnetic resonance; NASCET $=$ North American Symptomatic Carotid Endarterectomy Trial; PSV = peak systolic velocity; TOF = time-of-flight; $2 \mathrm{D}$ = two-dimensional; $3 \mathrm{D}=$ three-dimensional. logical deterioration in the form of a major or minor stroke related to acute thrombus or distal embolization.

This failed trial forced a major rethinking of the medical management and technological developments associated with CA stent implantation. During the next wave of CA stent trials, strict antiplatelet and antithrombotic regimens with clopidogrel, aspirin, and heparin were introduced. Even with advances in stent technology, morbidity rates were still 7 to $10 \%$; these rates were not significantly better than those associated with CEA for high-risk patients. ${ }^{13,25}$

This experience led to the era of distal embolic protection devices. The use of these devices has lowered the complication rates of CA stent placement procedures significantly (trials involving distal embolic protection devices will be discussed in detail later). Although the first report of distal embolic protection for CA revascularization was published by Theron, et al., ${ }^{31}$ in 1990, it was not until a decade later that investigators conducting CA stent trials began to embrace the utility of these devices in preventing procedure-related stroke.

\section{CLINICAL PROTOCOLS}

\section{Patient Selection}

Now that both surgical and endovascular alternatives exist for patients with CA occlusive disease, optimal pa- 
tient selection for each procedure will result in the lowest morbidity rates and the most favorable outcomes. Most pivotal trials of CA stent implantation have focused on both symptomatic and asymptomatic CA disease in patients who are considered to be high-risk surgical candidates. These high-risk characteristics have been previously defined in large surgical series, such as the NASCET and the Asymptomatic Carotid Atherosclerosis Study. ${ }^{10,23}$ These characteristics include anatomical ones (restenosis after CEA, contralateral occlusion, previous neck radiation or surgery, surgically inaccessible lesions [for example, located above the C-2 level or below the clavicle], neck immobility, tracheostomy, contralateral laryngeal palsy, bilateral severe stenotic lesions requiring treatment, and severe intracranial stenosis) and medical comorbidities (unstable angina, poor cardiac ejection fraction, congestive heart failure, planned coronary artery bypass operation, obstructive pulmonary disease, and advanced age [ $>75$ or 80 years, depending on the trial]).

Recently acquired data have been found to demonstrate a role for CA stent placement in patients seen in common daily practice. The investigators in the Carotid Revascularization using Endarterectomy or Stenting Systems trial examined the treatment results in a low-risk patient population. ${ }^{8}$ In this nonrandomized prospective study, 300 patients who underwent CEA and 150 who underwent stent placement procedures were enrolled at a total of 20 sites. In patients in whom the GuardWire Plus embolic protection device (Medtronic Vascular, Santa Rosa, CA) was used, there was a $2 \%$ composite rate of stroke, death, and MI for stent placement procedures, compared with a rate of 3\% for CEA. The Carotid Revascularization using Endarterectomy or Stenting Systems trial is one of the first ones to show a benefit from stent insertion accompanied by distal embolic protection over CEA for low-risk patients (although the benefit was not statistically significant).

It is possible to stratify these low-risk patients further into groups that would be better served with either CEA or angioplasty with stent implantation. Patients with heavily calcified plaques, excessively tortuous vessels, or ICAs with lumen diameters smaller than $3 \mathrm{~mm}$ may be better served by CEA. ${ }^{16,17,19}$ Suboptimal revascularization in heavily calcified plaques is common because the calcium makes these lesions refractory to balloon remodeling; loops and significant vessel tortuosity make the placement of a guide catheter in a stable position as well as filter and stent delivery difficult; lumen diameters smaller than 3 $\mathrm{mm}$ do not safely accommodate most distal protection devices. Alternatively, patients with high bifurcations (at or above the C-2 level) or morbid obesity may be better served with stent insertion. For most patients the procedures may be equivalent; patient choice will be the deciding factor.

\section{Imaging Modalities}

The inclusion of participants in these pivotal CA stent trials was dependent on the severity of the stenosis identified using either ultrasonography or angiography. For this reason, the following discussion of imaging modalities will focus primarily on ultrasonography, with brief mention of other noninvasive imaging tools that are applicable for the high-risk cohort of patients with CA disease who may have difficulty tolerating conventional DS angiography because of severe peripheral vascular, cardiopulmonary, or renal disease. Significant advances in technology over the last decade have made noninvasive imaging a powerful tool in the detection and surveillance of CA stenosis. Ultrasonography, CT angiography, and MR angiography all play an important role in CA imaging. Asymptomatic patients with carotid bruits, those with a history of transient ischemic attack or ischemic stroke, and symptomatic patients with evidence of embolic infarction on intracranial imaging should undergo CA imaging. ${ }^{20}$

Ultrasonography. Its low cost and wide availability make ultrasonography the first-line and most routinely used method of noninvasive diagnosis and surveillance for CA stenosis. A complete evaluation involves examining the CCAs, external CAs, ICAs, and vertebral arteries. Examinations of the CAs should be performed using gray-scale, color Doppler, and spectral Doppler ultrasonography. Gray-scale ultrasonography is used for plaque characterization, assessment of luminal diameter, and measurement of CA wall thickness. Color and/or power Doppler ultrasonography is used in the assessment of blood flow, identification of vessels, detection of plaque, and to measure CA wall thickness. The spectral Doppler modality is used to evaluate the severity of the stenosis. ${ }^{5}$

Plaque is characterized as homogeneous or heterogeneous by using gray-scale ultrasonography. Homogeneous plaque consists of dense, fibrous, laminated, connective tissue, and its echogenicity is comparable to that of muscle. Heterogeneous plaques pose the highest risk of stroke, and these lesions contain at least one hypoechoic area; such an area indicates intraplaque hemorrhage and/or lipid deposits. There is no reliable sonographic sign for plaque ulceration. ${ }^{5}$

As blood flows through vessels of narrowing diameters, its velocity increases proportionally. The PSV value and visualization of plaque on gray-scale ultrasonography are the main parameters in diagnosing and classifying CA stenosis. ${ }^{5,30}$ The use of ultrasound measurement of flow velocities to grade stenoses in the CCA and ICA is more reliable than gray-scale or color Doppler imaging alone. 5,20,30 When multiple stenoses are present (tandem lesions), the one with the greatest luminal narrowing determines the extent of hemodynamic compromise.

In certain situations, the measurement of PSV in the ICA may not adequately reflect the severity of disease. Among the conditions that can lead to misrepresentation of the PSV as an indication of stenosis severity are the presence of tandem lesions, discrepancy between gray-scale plaque visualization and PSV in the ICA, increased CCA velocities, hypertension, hyperdynamic cardiac state, contralateral occlusion, or low cardiac output. ${ }^{11,30}$ In such cases, determination of the ICA/CCA PSV ratio and ICA end-diastolic velocity will provide further insight into the severity of the disease.

Considerable variability exists in the reported sensitivity and specificity of ultrasonography, compared with angiography, for accurately grading ICA stenosis. $7,20,24,30 \mathrm{Ad}-$ ditionally, the criteria for reporting and stratifying ICA stenosis vary among institutions. ${ }^{29,30}$ In 2003 , the Society of Radiologists in Ultrasound published recommendations 
for using ultrasonography in the diagnosis and interpretation of atherosclerotic stenosis of the ICA. ${ }^{30}$ The panel found that this modality is not effective in stratifying lesions with less than $50 \%$ stenosis; thus, they recommended that these lesions be classified in a single category (" $<$ $50 \%$ stenosis"). After analyzing the literature, the panel developed ultrasonography thresholds for the stratification of ICA stenosis (Table 1).

By using a PSV of $230 \mathrm{~cm} / \mathrm{second}$ for the ICA, investigators from Harvard ${ }^{7}$ determined that ultrasonography had a sensitivity of $79 \%$, specificity of $86 \%$, and accuracy of $84 \%$ for detecting 70 to $99 \%$ stenosis according to NASCET angiographic criteria. ${ }^{23}$ In a recent multicenter study consisting of 350 patients, the sensitivity of ultrasonography in diagnosing 70 to $99 \%$ stenosis according to the NASCET angiographic criteria was between 87.5 and $98.6 \%$, and the specificity was between 59.2 and $75 \% .{ }^{24}$ In another recent study, it was determined from a review of the literature that ultrasonography can accurately detect ICA stenosis of greater than $50 \%$ and that this modality has up to $98 \%$ sensitivity in detecting 70 to $99 \%$ stenosis according to NASCET angiographic criteria. ${ }^{20}$ Ultrasonography is not as sensitive in detecting angiographically confirmed stenosis that is less than $50 \%$ according to NASCET criteria.

The presence of tandem lesions will cause discordance between the gray-scale ultrasonography and spectral Doppler findings, such that the more downstream stenosis, if more severe, will dampen the PSV between the two lesions. ${ }^{27,30}$ When a tandem lesion is suspected, a second noninvasive imaging modality or conventional angiography should be used for complete evaluation. If a tandem lesion is located within the intracranial segments of

TABLE 1

Ultrasonography thresholds for stratifying ICA stenosis*

\begin{tabular}{lc}
\hline \hline \multicolumn{1}{c}{ Ultrasound Criteria } & Stenosis Classification \\
\hline PSV $<125 \mathrm{~cm} / \mathrm{sec}$; no plaque or intimal & normal \\
thickening visible (additional supporting & \\
criteria: ICA/CCA PSV ratio $<2.0$ & \\
$\&$ ICA EDV $<40 \mathrm{~cm} / \mathrm{sec}$ ) & $<50 \%$ \\
ICA PSV $<125 \mathrm{~cm} / \mathrm{sec}$; plaque \&/or intimal & \\
thickening visible $($ additional supporting & \\
criteria: ICA/CCA PSV ratio $<2.0$ & $50-69 \%$ \\
$\&$ ICA EDV $<40 \mathrm{~cm} / \mathrm{sec}$ ) & \\
PSV $125-230 \mathrm{~cm} / \mathrm{sec}$ plaque visible & \\
(additional supporting criteria: & $\geq 70 \%$ \\
ICA/CCA PSV ratio $2.0-4.0$ & \\
$\&$ ICA EDV $40-100 \mathrm{~cm} / \mathrm{sec})$ & \\
PSV $>230 \mathrm{~cm} / \mathrm{sec}$; gray-scale evaluation & \\
reveals plaque $\&$ luminal narrowing & \\
(additional supporting criteria: ICA/CCA & \\
PSV ratio $>4$ \& ICA EDV $>100 \mathrm{~cm} / \mathrm{sec})$ & near occlusion \\
color Doppler ultrasound evidence of a & \\
markedly narrowed lumen & \\
patent lumen on gray-scale evaluation; & \\
no flow detected on color, spectral, & \\
$\&$ power Doppler ultrasonography & \\
(MR, CT, or catheter angiography & \\
should be performed for confirmation) &
\end{tabular}

* Stratifications adapted from Spencer, et al. Abbreviation: EDV = enddiastolic velocity. the ICA and only the extracranial ICA is examined, the amount of CA stenosis will be underestimated. ${ }^{27}$

Pseudoocclusion occurs when color and spectral Doppler imaging fail to detect flow, yet there is a very narrow residual lumen. ${ }^{27}$ This apparent occlusion results from the markedly decreased flow velocities through the residual lumen. Improper adjustment of various ultrasound parameters (such as color gain, pulse repetition frequency, and filter) may result in misdiagnosis.

Pseudonormalization of PSV may occur when a highgrade stenosis worsens. ${ }^{27}$ At approximately 60 to $70 \%$ stenosis, blood flow reaches its peak velocity. Shortly thereafter, flow velocities rapidly decrease to zero. ${ }^{5}$ Velocity falloff typically occurs at a residual lumen diameter of less than $0.7 \mathrm{~mm}$. Overestimation of the degree of CA stenosis will occur if there is a high-grade contralateral narrowing. Significant stenosis of the CCA or ICA can cause an increase in PSV contralaterally. ${ }^{27}$

The evaluation of CA stenosis with ultrasonography is very user-dependent. Operator errors in positioning of the Doppler gate, using a Doppler angle exceeding $60^{\circ}$, incorrect positioning of the sample volume, and failure to visualize the distal extent of the plaque will lead to incorrect classification of CA stenosis.,30 Poor patient positioning will also confound an examination. Additionally, there exists considerable variability in the technical aspects of ultrasound machines among different manufacturers. Discrepancies in measurement can also occur between newer and older equipment.

According to the Society of Radiologists in Ultrasound, patients with at least $50 \%$ ICA stenosis who have no surgical history and who are candidates for prophylactic revascularization should undergo follow-up evaluations every 6 to 12 months. ${ }^{30}$ Patients with visible plaques, risk factors for thromboembolic neurovascular disease, and less than $50 \%$ stenosis should undergo follow-up review with ultrasonography every 1 to 2 years. A follow-up duration of 3 to 5 years is recommended for those with normal results on $\mathrm{CA}$ ultrasonography and known risk factors.

Even under ideal conditions, ultrasonography evaluation of the CA system will overestimate the degree of stenosis when compared with conventional angiography. ${ }^{24,29}$ Nevertheless, ultrasonography is considered to be a costeffective, accurate, first-line noninvasive examination for evaluation of CA stenosis. ${ }^{24}$ The advantages of ultrasonography are its low cost, wide availability, and zero complication risk, features making it an ideal method for the evaluation of CA stenosis. Furthermore, numerous studies show that ultrasonography is accurate, sensitive, and specific for diagnosis and screening of high-grade CA stenosis. $5,7,20,24$

The MR Angiography Modality: Basic Principles of Flow-Related Enhancement. The dipoles of protons in a magnetic field are manipulated to acquire a signal and ultimately an image. Alignments and manipulations of these proton dipoles are performed in different slice thicknesses within an external magnetic field by grading the field to align the protons to different degrees along the length of the magnet. This allows slice-by-slice information to be obtained. All the stationary manipulated protons in one slice would remain manipulated, whereas the flow- 
ing protons would move out of the slice, depending on the time for acquisition of this information. This is the principle behind TOF imaging. All information in a slice would consist of characteristic signals, whereas the flowing protons would be devoid of signal as the manipulated protons moved out of the slice and nonmanipulated ones moved into it.

Time-of-Flight MR Angiography. The most clinically applicable of the nonenhanced MR imaging modalities is TOF imaging. ${ }^{20}$ Both $2 \mathrm{D}$ and $3 \mathrm{D}$ TOF imaging of vessels can be performed. In the 2D TOF method, one anatomical slice is imaged at a time. The advantages of this process are extended coverage, shorter acquisition time, and better slow-flow sensitivity. Slow-flow sensitivity makes 2D TOF imaging optimal for venous studies. The disadvantages are the decreased signal-to-noise ratio, thicker slices, in-plane turbulence, overestimation of stenosis from dephasing, poor background suppression of fat, and misregistration artifact. In the 3D TOF method, information is acquired from a volumetric slice of tissue and then partitioned into thinner sections. The advantages of this method are the thinner slices, higher signal-to-noise ratio, and decreased susceptibility to flow artifacts. The disadvantages are decreased sensitivity to slow flow, saturation signal loss, patient motion artifact, and longer imaging times (R Alberico, personal communication, 2004).

Gadolinium-Enhanced or Contrast-Enhanced MR Angiography. Although it has proved to be impractical to quantify CA stenosis accurately with either TOF or contrast-enhanced MR angiography, the latter method shows promise for distinguishing higher-grade stenosis., ${ }^{9,22}$ The advantages of contrast-enhanced MR angiography are that it lacks the inherent limitations of imaging flow with related artifacts. There is less susceptibility and motion artifact and better spatial resolution. The disadvantages consist of the need for close attention to timing, the loss of directional flow data, and the need for improved gradients ( $\mathrm{R}$ Alberico, personal communication, 2004). There is also the problem of the contrast agent itself with respect to related reactions and the need for intravenous access.

When contrast-enhanced MR angiography is performed in conjunction with ultrasonography, the diagnostic accuracy in determining the percentage of stenosis is dramatically increased. ${ }^{4,15,32}$ When MR angiography and ultrasonography are combined, the sensitivity and specificity in detecting significant CA stenosis approach 96 and $80 \%$, respectively; however, adding MR angiography increases costs significantly. ${ }^{7}$ Some investigators have suggested that the combined modality approach could eliminate the need for preoperative conventional catheter angiography. ${ }^{28}$ Still, the current consensus is that DS angiography is the gold standard for the measurement of CA stenosis, ${ }^{26}$ for reasons to be discussed later.

The CT Angiography Modality. Technological advances, including multidetector scanners, improved software, and newer volume-rendering techniques make imaging faster and more reliable. The CT angiography modality may eventually supplant DS angiography for cases in which there are discordant findings on contrast-enhanced MR angiography and ultrasonography. ${ }^{20}$ The advantages of CT angiography are many. First, this modality is an anatomically dependent one that is relatively insensitive to the flow-related problems encountered with MR angiography, thus resulting in less overestimation of stenosis, and CT angiography also depicts calcification reliably. Many patients with CA disease have heavy calcification, which is difficult to treat adequately with stent-assisted angioplasty. Also, CT angiography can be used to demonstrate tandem stenosis and to document the course of a vessel that has low or absent flow. In addition to the requirement of contrast medium, a disadvantage of CT angiography compared with MR angiography is the technical factor. Significant attention must be given to processing details and meticulous technique must be adhered to in producing quality images for interpretation.

Clinical Utility of DS Angiography. The utility of angiography for interventional procedures remains, quite simply, the ability that it provides to understand the vasculature leading toward and arising beyond the lesion. For $\mathrm{CA}$ intervention, exquisite knowledge of the relevant proximal and distal vasculature is imperative. Understanding the aortic arch anatomy is vital to deciding which delivery apparatus can be used in the stent placement procedure. For example, if there is excessive aortic arch atheroma or corkscrew tortuosities at the origins of the left CCA or innominate artery, the patient may be better served with a CEA. Additionally, if there are proximal stenoses at the CA origins or in the iliac vessels, considerations of whether these lesions require treatment to facilitate CA stent delivery need to be addressed.

Alternatively, understanding the intracranial anatomy is vital to selecting distal embolic protection devices. In the event of an isolated middle cerebral artery or an incompetent circle of Willis, a filter type of protection device might be preferred to an occlusive type. In the event of coexistence of an intracranial tandem lesion, this lesion might be addressed before revascularization of the extracranial one is performed. Understanding the pathways to the target lesion (in this case the CA) well before the procedure allows clinicians to select the guide catheters, wires, and devices that will minimize the risks associated with CA intervention. As is the case in most surgical procedures, $90 \%$ of the intervention is accomplished in the planning phase, and only DS angiography currently provides all the necessary information required for effective planning of the intervention.

\section{Overview of Technique for Placement of Distal Protection Devices}

The three methods of distal protection most commonly used are filter protection, which allows passage of blood but not particles and debris (pore size of the filters $\sim 150$ $\mu \mathrm{m})$; balloon occlusion, which prevents the passage of blood and debris; and flow reversal, which causes retrograde flow in the CA that prevents the migration of debris into the intracranial circulation. The method for CA stent delivery has been described by our group and others previously. ${ }^{14}$ The following summary will focus on the additional steps required for the positioning and retrieval of a distal embolic protection device (filter type).

Delivery of Distal Embolic Protection Devices. Once the guide catheter or sheath is positioned in the CCA proximal to the stenotic lesion, a distal embolic protection de- 
vice (we prefer to use a retrievable filter) mounted on a 0.014-in microwire is carefully guided across the stenotic region by using the biplanar roadmapping technique. Distal embolic protection devices typically have shapable tips that allow the user to steer the device delicately through the stenotic plaque, avoiding traumatizing ulcerations. The device should be placed in a relatively straight segment of the distal ICA, called a "landing strip," and then unsheathed for release. Once the embolic protection device is deposited, its apposition to the vessel wall is assessed by obtaining at least two angiographic views of the retrievable filter.

After completing the angioplasty that is performed after stent placement, cervical and intracranial images are obtained to assess target vessel patency and to exclude evidence of any major intracranial vessel occlusion. Once this is done, the protection filter device is recaptured. This is accomplished by advancing a retrieval sheath over the wire of the filter and then engulfing the device slowly, preventing the extrusion of debris during removal of the filter from the body. The course of the retrieval sheath through the segment of stent-treated vessel should be carefully observed because the retrieval sheath may catch on the struts protruding from the stent (this is more common with open-cell stents). If this occurs, advancing the guide sheath closer to or through the stent may provide enough support to allow the stent to be crossed with the retrieval sheath. Substituting the retrieval sheath for this catheter may allow the operator to navigate its tip around the difficult stent segment.

When distal balloon occlusion is used to provide embolic protection, $60 \mathrm{ml}$ of blood (and debris) is aspirated before the balloon is deflated. The aspiration is accomplished through an export catheter placed just proximal to the balloon.

\section{Outcomes of CA Stent Placement in the Era of Distal Protection}

Distal protection devices may be considered the single technological advancement that has reduced complication rates after CA stent placement to levels below those of CEA. ${ }^{8}$ The filter-protection method is used in most contemporary CA stent trials.

The first National Institutes of Health-sponsored study in which CEA was compared with stent placement in conjunction with filter protection in high-risk patients was the CREST investigation. Data from the credentialing phase demonstrated a composite stroke and death rate of $3.4 \%$ for both asymptomatic and symptomatic patients (527 individuals) (G Roubin for the investigators: oral presentation, American Stroke Association Meeting, November 9-12, 2003, Orlando, FL). Although this morbidity rate was markedly lower than that reported in previous trials in which distal protection devices were not used, it was expected that the complication rate would continue to decrease as clinicians gained experience. In addition to the CREST, there have been six pivotal CA stent trials in which various stents and distal protection devices were used (Table 2).

In addition to examining periprocedural morbidity and mortality levels, questions concerning the durability of CA stents may preclude the use of these devices in the
TABLE 2

Pivotal CA stent trials

in which distal embolic protection was used*

\begin{tabular}{|c|c|c|}
\hline $\begin{array}{c}\text { Trial } \\
\text { (manufacturer/sponsor) }\end{array}$ & Stent & $\begin{array}{l}\text { Distal Protection } \\
\text { Device }\end{array}$ \\
\hline $\begin{array}{l}\text { ARCHeR Phases } 2 \& 3 \\
\text { (Guidant) }\end{array}$ & $\begin{array}{l}\text { ACCULINK } \\
\text { (rapid-exchange } \\
\text { version in Phase 3) }\end{array}$ & $\begin{array}{l}\text { ACCUNET } \\
\text { (rapid-exchange } \\
\text { version in Phase 3) }\end{array}$ \\
\hline $\begin{array}{l}\text { BEACH } \\
\text { (Boston Scientific) }\end{array}$ & monorail Wallstent & EPI FilterWire EZ \\
\hline $\begin{array}{l}\text { CABERNET } \\
\text { (Boston Scientific \& } \\
\text { EndoTex) }\end{array}$ & NexStent & $\begin{array}{l}\text { EPI FilterWire } \\
\text { EX/EZ }\end{array}$ \\
\hline $\begin{array}{l}\text { MAVEriC } \\
\text { (Medtronic AVE) }\end{array}$ & AVE & PercuSurge \\
\hline $\begin{array}{l}\text { SAPPHIRE } \\
\text { (Cordis) }\end{array}$ & Smart or Precise & $\begin{array}{l}\text { Angioguard or } \\
\text { Angioguard XP }\end{array}$ \\
\hline $\begin{array}{l}\text { SECuRITY } \\
\text { (Abbott) }\end{array}$ & X-Act & $\begin{array}{l}\text { previously } \\
\text { Neuroshield, now } \\
\text { EmboShield }\end{array}$ \\
\hline
\end{tabular}

* Table adapted from Levy EI, Kim SH, Bendok BR, et al: Interventional Neuroradiologic Therapy, in Mohr JP, Choi DW, Grotta JC, et al (eds): Stroke: Pathophysiology, Diagnosis, and Management, ed 4. Philadelphia: Churchill Livingstone (An Imprint of Elsevier), 2004, pp 14751520. Abbreviations: ARCHeR = ACCULINK for Revascularization of Carotids in High-Risk Patients; MAVEriC = Evaluation of the Medtronic AVE Self-Expanding Carotid Stent System with Distal Protection in the Treatment of Carotid Stenosis; SAPPHIRE $=$ Stenting and Angioplasty with Protection in Patients at High Risk for Endarterectomy; SECuRITY = The Registry Study to Evaluate the Neuroshield Bare Wire Cerebral Protection System and X-Act Stent in Patients at High Risk for Carotid Endarterectomy.

younger population. In the ACCULINK for Revascularization of Carotids in High-Risk Patients trial, target lesion revascularization was specifically examined at 6 months and 1 year after stent implantation. ${ }^{12}$ Repeated revascularization (angioplasty, stent insertion, CEA, or thrombolysis) for recurrent stenosis (angiographic evidence of $>$ $80 \%$ stenosis in asymptomatic or $>50 \%$ stenosis in symptomatic patients were the criteria used to determine which individuals required repeated treatment) in Phase 1 of this trial resulted in a mean rate of target lesion revascularization of $0.7 \%$ at 6 months and $2.2 \%$ at 1 year. ${ }^{33}$ Revascularization rates after CA stent placement appear to be within this range. In another study consisting of 112 patients (117 vessels) with a mean Doppler follow-up duration of 16 months (range 4-49 months), $80 \%$ or greater restenosis was detected within the stent in six patients $(5 \%) .^{18}$ Target lesion revascularization was accomplished in $5 \%$ of the patients treated for recurrent symptoms. These rates are comparable with those for CEA.

The interim results of The Registry Study to Evaluate the Neuroshield Bare Wire Cerebral Protection System and X-Act Stent in Patients at High Risk for Carotid Endarterectomy were presented in 2003 (P Whitlow for the investigators: oral presentation, The Registry Study to Evaluate Neuroshield Bare Wire Cerebral Protection System and X-Act Stent in Patients at High Risk for Carotid Endarterectomy, Transcatheter Therapeutics Meeting, September 17, 2003, Washington, DC). Potential embolic debris was identified in $89 \%$ of the filters examined after removal from the patients treated. This finding requires us seriously to consider whether CA stent-assisted 
revascularization should be performed without distal protection. The combined major and minor stroke rate at 30 days was $6.9 \%$, and the combined death and stroke rate was $7.2 \%$.

Of the recent pivotal CA stent studies, the Stenting and Angioplasty with Protection in Patients at High Risk for Endarterectomy trial is one of the few in which patients have been randomized to groups receiving either stent implantation with the Angioguard or Angioguard XP distal protection device (Cordis, Miami Lakes, FL) or CEA. ${ }^{34}$ In this study, patients who met eligibility criteria were evaluated by interventionists and surgeons, and only those deemed suitable for either therapy were randomized. Patients with stenotic lesions that were not equally suitable for either stent placement or CEA were entered in a stent or surgical registry. One hundred sixty-seven patients were randomly assigned to each treatment group (and an additional 406 patients were included in the stent registry).

At 30 days, the rates of death, myocardial infarction, or major adverse events were lower for patients who received stents than for those who underwent CEA, but the differences between the treatment groups did not reach statistical significance. Interestingly, there were stroke rates of $3.6 \%$ in the stent arm and 3\% in the surgical arm of the study. At 1 year, however, the rates of stroke, death, MI, and major adverse events were lower in the stent arm (but with no statistical significance). When examining the durability of stent treatment compared with CEA, the rates of target lesion revascularization were $0.6 \%$ in the stent arm and $3.6 \%$ in the surgical arm of the study $(p=0.12)$. Evidence of stenosis exceeding $80 \%$ was detected using ultrasonography in $0.8 \%$ of patients after stent implantation and in $4.2 \%$ of patients after surgery. The complications (which were statistically significant in favor of stent procedures) were cranial nerve palsy (which is unlikely after stent placement) and periprocedural MI $(\mathrm{p}=0.04)$. At 1 year, there were no major ipsilateral strokes after stent procedures, but the incidence was 3\% (five cases) after CEA $(p=0.03)$.

The Stenting and Angioplasty with Protection in Patients at High Risk for Endarterectomy trial provided evidence that stent implantation in high-risk patients with CA disease is not inferior to CEA. This finding has led to the recent approval by the US Food and Drug Administration of CA angioplasty and stent implantation with the ACCUNET and ACCULINK devices (Guidant Corp., Santa Clara, CA) in high-risk surgical patients (accessed November 18, 2004: http://www.fda.gov/cdrh/pdf4/ p040012a.pdf).

The BEACH study was designed as a single-arm registry to evaluate the benefits of the EPI FilterWire distal embolic protection device (Boston Scientific, Natick, MA) used in conjunction with the Wallstent (Boston Scientific) (LN Hopkins for the investigators, Universal Cerebral Protection: BEACH Trial Results with the Carotid Wallstent for Carotid Artery Stenting in High-Risk Surgical Patients, oral presentation, Transcatheter Therapeutics 2004, September 29, 2004, Washington, DC). High-risk surgical candidates (747 patients) with CA stenosis of $50 \%$ or greater in symptomatic and $80 \%$ or greater in asymptomatic patients were enrolled. Some of the main advantages of the devices used in this trial were that all were monorail (or rapid-exchange) devices that fit through a No. 6 French guide catheter. This provided a significant advantage of flexibility, compared with No. 6 French guiding sheaths, allowing for greater ease and rapidity in the placement of guide catheters just proximal to target lesions. Additionally, the FilterWire is a highly navigable distal protection filter that can be delivered despite the inherent tortuosities and kinks of the vasculature, which often prevent the delivery of other filter protection devices. The increased flexibility of the entire system may have contributed to the overall low (3.1\%) 30-day rate of ipsilateral major or minor stroke. The composite 30-day complication rate, which includes both Qwave and non-Q-wave MIs, was 5.4\%.

The CABERNET is one of the most recent single-arm registries for which results have been reported (LN Hopkins for the investigators: CABERNET - 30-day Trial Results from the Use of a Novel Carotid Stent and Embolic Protection System, oral presentation, Transcatheter Therapeutics, September 29, 2004, Washington, DC). This study was designed as a prospective registry for evaluation of the outcomes in patients with CA stenosis who were at high risk for requiring CEA after stent-assisted revascularization with adjunctive distal protection. The stent used in this study (NexStent; EndoTex Interventional Systems, Cupertino, CA [now owned by Boston Scientific]) has a unique feature in that it is made of a rolled sheet of nitinol (a nickel-titanium alloy) that unrolls to conform exactly to the lumen of the target vessel. Additionally, the stent is highly flexible and has a closed-cell design, which is of benefit when using distal protection devices because retrieval catheters are less likely to get caught on stent tines when attempting to recapture the filter.

This study differed from its predecessors in that patients with asymptomatic lesions with $60 \%$ stenosis were included. Additionally, lesions could not be longer than 30 $\mathrm{mm}$, thus limiting enrollment to more focal lesions. Of the 443 patients evaluated at the 30-day end point of the trial, approximately $75 \%$ had been treated initially for asymptomatic lesions. The 30-day composite rate of stroke, death, and MI at the end point of the study was $3.8 \%$, the lowest reported to date. Several factors likely accounted for this result: study centers were carefully selected on the basis of surgeons' experience in performing CA stent placement, asymptomatic lesions with $60 \%$ stenosis were included in the trial, and only lesions $30 \mathrm{~mm}$ or less in length that could be treated with a single stent were included in the trial. Additionally, by the start of this trial, clinicians had become aware of criteria that could be used in patients with CA stenosis to tell who was suited to stent implantation and who was not (for example, patients with heavily calcified plaques, excessive tortuosity of the CA, and difficult arch anatomy were unsuitable candidates).

\section{CONCLUSIONS}

The combination of increasing clinician experience, better pharmacological agents, and distal protection devices appears to have lowered the stroke rates for highrisk patients undergoing endoluminal CA revascularization to approximately those for low-risk patients under- 
going CEA (that is, $<3 \%$ for asymptomatic lesions ${ }^{10}$ and $<6 \%$ for symptomatic lesions ${ }^{23}$ ). The question remains: is distal protection for everyone? The answer, although seemingly simple, depends on many variables. Among those variables are ICA anatomy and the surgeon's familiarity with distal protection devices. These devices introduce additional steps into the stent placement procedure and require a familiarity with these steps and with the limitations associated with the use of these devices. Additionally, distal protection devices may induce some vasospasm, or it may be impossible to navigate them across tight kinks in the CA.

Despite these concerns, we recommend the routine use of distal protection devices in conjunction with CA stent implantation to prevent the migration of embolic debris into the intracranial circulation. Although the use of distal protection devices has not been investigated in a large multicenter trial in which patients have been randomized to receive protected or unprotected stents, the results in the trials described here are superior to those in CA stent trials conducted before distal protection was in use. Is this treatment for everyone? Clearly, stent placement procedures have shown superiority over CEA for the select cohort of high-risk surgical patients; without a doubt, however, there are patients for whom CEA is the preferable intervention. As clinicians, the better we can stratify patients who are ideally suited for stent placement or CEA, the more likely we are to optimize outcomes while minimizing procedure-related morbidity.

\section{Disclosure}

Dr. Hopkins receives research support from and is a consultant for the Boston Scientific, Cordis, EndoTex, Guidant, and Medtronic companies. He has a financial interest in EndoTex. Drs. Levy and Hanel are consultants for Cordis Corporation and have received educational grants from Cordis and Boston Scientific Corporations.

\section{References}

1. Akiyama T, Moussa I, Reimers B, et al: Angiographic and clinical outcome following coronary stenting of small vessels: a comparison with coronary stenting of large vessels. J Am Coll Cardiol 32:1610-1618, 1998

2. Bergeron $\mathrm{P}$, Chambran $\mathrm{P}$, Benichou $\mathrm{H}$, et al: Recurrent carotid disease: will stents be an alternative to surgery? J Endovasc Surg 3:76-79, 1996

3. Bockenheimer SA, Mathias K: Percutaneous transluminal angioplasty in arteriosclerotic internal carotid artery stenosis. AJNR 4:791-792, 1983

4. Borisch I, Horn M, Butz B, et al: Preoperative evaluation of carotid artery stenosis: comparison of contrast-enhanced MR angiography and duplex sonography with digital subtraction angiography. AJNR 24:1117-1122, 2003

5. Brant WE: The Core Curriculum: Ultrasound. Philadelphia: Lippincott Williams \& Wilkins, 2001

6. Briguori C, Sarais C, Pagnotta P, et al: In-stent restenosis in small coronary arteries: impact of strut thickness. J Am Coll Cardiol 40:403-409, 2002

7. Buskens E, Nederkoorn PJ, Buijs-Van Der Woude T, et al: Imaging of carotid arteries in symptomatic patients: cost-effectiveness of diagnostic strategies. Radiology 233:101-112, 2004

8. CARESS Steering Committee: Carotid revascularization using endarterectomy or stenting systems (CARESS): phase I clinical trial. J Endovasc Ther 10:1021-1030, 2003

9. Enochs WS, Ackerman RH, Kaufman JA, et al: Gadolinium-enhanced MR angiography of the carotid arteries. J Neuroimaging 8:185-190, 1998

10. Executive Committee for the Asymptomatic Carotid Atherosclerosis Study: Endarterectomy for asymptomatic carotid artery stenosis. JAMA 273:1421-1428, 1995

11. Grant EG, Benson CB, Moneta GL, et al: Carotid artery stenosis: gray-scale and Doppler US diagnosis-Society of Radiologists in Ultrasound Consensus Conference. Radiology 229: 340-346, 2003

12. Gray WA: The ARCHeR Trials: Final One Year Results. (http://www.summerinseattle.com/home/archer.pdf) [Accessed 6 December 2004]

13. Gray WA, White HJ Jr, Barrett DM, et al: Carotid stenting and endarterectomy: a clinical and cost comparison of revascularization strategies. Stroke 33:1063-1070, 2002

14. Guterman LR, Fessler RD, Hopkins LN: Cervical carotid revascularization. Neurosurg Clin N Am 11:39-48, viii, 2000

15. Johnston DC, Eastwood JD, Nguyen T, et al: Contrast-enhanced magnetic resonance angiography of carotid arteries: utility in routine clinical practice. Stroke 33:2834-2838, 2002

16. Kerber CW, Cromwell LD, Loehden OL: Catheter dilatation of proximal carotid stenosis during distal bifurcation endarterectomy. AJNR 1:348-349, 1980

17. Koning R, Eltchaninoff H, Commeau P, et al: Stent placement compared with balloon angioplasty for small coronary arteries: in-hospital and 6-month clinical and angiographic results. Circulation 104:1604-1608, 2001

18. Levy EI, Hanel RA, Lau T, et al: Frequency and management of recurrent stenosis after carotid stent implantation. J Neurosurg 102:29-37, 2005

19. Moer R, Myreng Y, Molstad P, et al: Stenting in small coronary arteries (SISCA) trial. A randomized comparison between balloon angioplasty and the heparin-coated beStent. J Am Coll Cardiol 38:1598-1603, 2001

20. Morgan JA, Ackerman RH, Romero JM, et al: Noninvasive imaging evaluation of carotid artery occlusive disease. Appl Radiol 33:16-24, 2004

21. Naylor AR, Bolia A, Abbott RJ, et al: Randomized study of carotid angioplasty and stenting versus carotid endarterectomy: a stopped trial. J Vasc Surg 28:326-334, 1998

22. Nederkoorn PJ, Elgersma OE, van der Graaf Y, et al: Carotid artery stenosis: accuracy of contrast-enhanced MR angiography for diagnosis. Radiology 228:677-682, 2003

23. North American Symptomatic Carotid Endarterectomy Trial Collaborators: Beneficial effect of carotid endarterectomy in symptomatic patients with high-grade carotid stenosis. N Engl J Med 325:445-453, 1991

24. Patel MR, Kuntz KM, Klufas RA, et al: Preoperative assessment of the carotid bifurcation. Can magnetic resonance angiography and duplex ultrasonography replace contrast arteriography? Stroke 26:1753-1758, 1995

25. Qureshi AI, Knape C, Maroney J, et al: Multicenter clinical trial of the NexStent coiled sheet stent in the treatment of extracranial carotid artery stenosis: immediate results and late clinical outcomes. J Neurosurg 99:264-270, 2003

26. Remonda L, Senn P, Barth A, et al: Contrast-enhanced 3D MR angiography of the carotid artery: comparison with conventional digital subtraction angiography. AJNR 23:213-219, 2002

27. Romero JM, Lev MH, Chan ST, et al: US of neurovascular occlusive disease: interpretive pearls and pitfalls. Radiographics 22:1165-1176, 2002

28. Rothwell PM: For severe carotid stenosis found on ultrasound, further arterial evaluation prior to carotid endarterectomy is unnecessary: the argument against. Stroke 34:1817-1819, 2003

29. Sabeti S, Schillinger M, Mlekusch W, et al: Quantification of internal carotid artery stenosis with duplex US: comparative 
analysis of different flow velocity criteria. Radiology 232: 431-439, 2004

30. Spencer EB, Sheafor DH, Hertzberg BS, et al: Nonstenotic internal carotid arteries: effects of age and blood pressure at the time of scanning on Doppler US velocity measurements. Radiology 220:174-178, 2001

31. Theron J, Courtheoux P, Alachkar F, et al: New triple coaxial catheter system for carotid angioplasty with cerebral protection. AJNR 11:869-877, 1990

32. Townsend TC, Saloner D, Pan XM, et al: Contrast materialenhanced MRA overestimates severity of carotid stenosis, compared with 3D time-of-flight MRA. J Vasc Surg 38:36-40, 2003
33. Wholey M: ARCHeR (Acculink for Revascularization of Carotids in High-Risk Patients). Clin Cardiol 26:296, 2003 (Abstract)

34. Yadav JS, Wholey MH, Kuntz RE, et al: Protected carotidartery stenting versus endarterectomy in high-risk patients. $\mathbf{N}$ Engl J Med 351:1493-1501, 2004

Manuscript received November 23, 2004.

Accepted in final form December 4, 2004.

Address reprint requests to: Elad I. Levy, M.D., Department of Neurosurgery and Toshiba Stroke Research Center, University at Buffalo, 3 Gates Circle, Buffalo, New York 14209. email: elevy @buffns.com. 